\title{
Tractable Algorithm for Robust Time-Optimal Trajectory Planning of Robotic Manipulators under Confined Torque
}

\author{
Q. Zhang, S.-R. Li, J.-X. Guo, X.-S. Gao
}

\author{
Qiang Zhang, Shu-Rong Li \\ Automation Department \\ China University of Petroleum(East China) \\ E-mail:zhangqiangupc@gmail.com, lishuron@upc.edu.cn
}

Jian-Xin Guo, Xiao-Shan Gao*

Academy of Mathematics and Systems Science

Chinese Academy of Sciences

E-mail:guojianxin@amss.ac.cn

*Corresponding author: xgao@mmrc.iss.ac.cn

\begin{abstract}
In this paper, the problem of time optimal trajectory planning under confined torque and uncertain dynamics and torque parameters along a predefined geometric path is considered. It is shown that the robust optimal solution to such a problem can be obtained by solving a linear program. Thus a tractable algorithm is given for robust time-optimal path-tracking control under confined torque.

Keywords: robust optimal control, time minimum trajectory planning, parameter uncertainties, tractable algorithm.
\end{abstract}

\section{Introduction}

In order to maximize productivity, the problem of minimum time motion planning of robotic manipulators is widely studied. In practical applications, the motion planning problem can be solved by two kinds of methods: the coupled method and the decoupled method [1]. As the name implies, the coupled methods determine the geometric path and the velocity along the path simultaneously. On the other hand, the decoupled methods determine the geometric path first according to certain criteria, and then determine the velocity along the path to minimize the path traversal time. This paper follows the decoupled approach due to the following reasons. Simultaneous optimization of the geometric path and the velocity is computationally difficult [2], especially when one wants to find a tractable algorithm. Also, in many industrial applications, the geometric path is predefined and does not need to be optimized.

Many efficient approaches for the time minimum trajectory planning (TMTP) problem along a predefined geometric path have been proposed, including the phase plane analysis approaches [3-6], the Pontryagin maximum principle and shooting algorithms [7], the convex optimization $[2,10]$, the path reshaping [8], and the direct search method [9]. The recent works $[2,11]$ are significant in which general and efficient methods are proposed. In [2], the second-order cone program is applied to solve the corresponding convex optimization problem. In [11], the sequential linear programming is used to solve the TMTP, which has better computational complexity than that of the phase plane method given in [4].

The above approaches assume that the dynamics model of the robotic manipulator is precisely known and equal to some nominal values. In practice, the dynamic model always has uncertainties. For example, to simplify the solution procedure, the viscous friction is often neglected and the uncertainties caused by the payload variation are often ignored. Many robust trajectory planning methods were proposed to handle these uncertainties. One of the commonly used approach is the min-max optimization approach which obtains the time minimum solution in the case of worst disturbances [12-14]. Due to its bi-level structure, min-max optimization problems pose 
challenges for efficient numerical solution in many cases. Other interesting approaches include the stochastic optimization approach [15] and the predictive control $[16,17]$.

In this paper, a tractable offline algorithm is given for the robust TMTP of robotic manipulators under torque limits and bounded dynamic parameter uncertainties and torque disturbances based on the min-max optimization approach. To solve the problem, it is shown that the TMTP problem for robotics under confined torque is equivalent to a linear optimal control problem meaning that both the objective function and the constraints are linear in the control and state variables. When disturbances are added, a robust linear optimal control problem is obtained, which is formulated as a min-max optimization problem. Furthermore, it is shown that when the trajectory is approximated with a quadratic B-spline curve, the min-max optimization problem is reduced to a min-max linear optimization problem which in turn can be reduced to a plain linear programming problem and thus is tractable, meaning that the problem can be solved in polynomial time. Numerical simulations are used to show that the method gives high precision approximate solutions to the robust TMTP very efficiently.

Comparing to existing approaches [12-14], the proposed method seems to be the first to convert the robust TMTP for robotics into a linear program. Linear program was also used in [12], where the objective function is not equivalent to time-minimality.

The rest of the paper is organized as follows. In Section 2, the nominal TMTP problem is reduced to a linear control problem. In Section 3, methods of parametrization is given. In Section 4, a tractable algorithm for the robust TMTP problem is given. In Section 5, numerical examples are presented. Conclusions are presented in Section 6.

\section{Linear Formulation for TMTP under Confined Torque}

In this section, we will show that the TMTP problem can be formulated as a linear optimal control problem meaning that the objective function and the constraints of the problem are linear in the control and state variables.

Consider an $n$ DOF robotic manipulator satisfying the following dynamic equation [2]

$$
\tau=\mathbf{M}(\mathbf{q}) \ddot{\mathbf{q}}+\dot{\mathbf{q}}^{T} \mathbf{C}(\mathbf{q}) \dot{\mathbf{q}}+\mathbf{G}(\mathbf{q}),
$$

where $\tau \in \mathbf{R}^{n}$ is the vector of actuator torques, $\mathbf{q} \in \mathbf{R}^{n}$ is the vector of joint angular positions, $\mathbf{M}(\mathbf{q}) \in \mathbf{R}^{n \times n}$ is the inertia matrix of the manipulator, $\mathbf{C}(\mathbf{q}) \in\left(\mathbf{R}^{n}\right)^{n \times n}$ is a matrix, whose elements are in $\mathbf{R}^{n}$, representing the coefficients of the centrifugal and Coriolis forces, and $\mathbf{G}(\mathbf{q}) \in$ $\mathbf{R}^{n}$ is the vector of gravitational torques.

In this paper, the given path to be tracked in task space is denoted by

$$
\mathbf{r}(s)=[x(s), y(s), z(s)]^{\mathrm{T}}, s \in[0,1],
$$

and the corresponding joint path $\mathbf{q}(s)$ in the joint space is also assumed to be determined by considering task requirement, obstacle avoidance, singularity avoidance, etc. Hence, a kinematics map between $\mathbf{r}(s)$ and $\mathbf{q}(s)$ exists. Here the path singularity problem is not considered since it can be avoided by properly adjusting the joint path.

Following [4], each element of $\mathbf{r}(s)$ is assumed to be a piecewise analytic real-valued function and has at least $C^{1}$ continuity at the connection points. Furthermore, $\mathbf{r}$ is assumed to be nonsingular, that is, for any $s \in[0,1]$ there exists at least one $l \in\{x, y, z\}$ such that $\frac{\mathrm{d} l(s)}{\mathrm{d} s} \neq 0$.

Denote $1=\frac{\mathrm{d}}{\mathrm{d} s}$ and ${ }^{\cdot}=\frac{\mathrm{d}}{\mathrm{d} t}$, where $t$ represents time. Then the joint velocity and joint acceleration can be formulated as

$$
\dot{\mathbf{q}}=\mathbf{q} / \dot{s}, \quad \ddot{\mathbf{q}}=\mathbf{q} / \ddot{s}+\mathbf{q} / / \dot{s}^{2} .
$$


Following (1) and (3), the dynamics model can be written as a linear function in $\ddot{s}$ and $\dot{s}^{2}$ :

$$
\tau=\mathbf{m}(s) \ddot{s}+\mathbf{c}(s) \dot{s}^{2}+\mathbf{g}(s),
$$

where $\mathbf{m}(s)=\mathbf{M}(\mathbf{q}(s)) \mathbf{q} \prime(s) \in \mathbf{R}^{n}, \mathbf{c}(s)=\mathbf{M}(\mathbf{q}(s)) \mathbf{q} \prime \prime(s)+\mathbf{q} /(s)^{T} \mathbf{C}(\mathbf{q}(s)) \mathbf{q} \prime(s) \in \mathbf{R}^{n}$, $\mathbf{g}(s)=\mathbf{G}(\mathbf{q}(s)) \in \mathbf{R}^{n}$.

The TMTP problem can be described as the following optimal control problem [2]:

$$
\begin{array}{ll} 
& \min _{b(s)} J_{T}=\int_{0}^{1} \frac{1}{\sqrt{a(s)}} \mathrm{d} s \\
\text { s.t. } & a \prime(s)=2 b(s), \forall s \in[0,1], \\
& a(s)>0, \forall s \in(0,1), a(0)=a_{0}, a(1)=a_{1}, \\
& \mathbf{m}(s) b+\mathbf{c}(s) a+\mathbf{g}(s)=\tau(s), \\
& \tau_{\min } \leq \tau(s) \leq \tau_{\max },
\end{array}
$$

where $a=\dot{s}^{2}, b=\ddot{s}, \tau_{\min }$ and $\tau_{\max }$ are the bound vectors for the torque, and the boundary values $a(0)$ and $a(1)$ can be obtained from (3) and the boundary values of the joint velocities: $\dot{\mathbf{q}}(0)=\dot{\mathbf{q}}_{0}, \dot{\mathbf{q}}(1)=\dot{\mathbf{q}}_{\mathrm{f}}$. When $b(s)=\ddot{s}$ is known, $\dot{s}$ and $\mathbf{q}(t)$ can be uniquely determined.

Problem (5) has the following important property.

Theorem 1. The optimal solution $\dot{s}_{o}(s), s \in[0,1]$ of problem (5) is unique and is maximum at any parameter value. That is, let $\dot{s}_{f}(s)$ be another parameter velocity satisfying the constraints of (5). Then, $\dot{s}_{f}(s) \leq \dot{s}_{o}(s)$ for all $s \in[0,1]$.

Theorem 1 follows from the proof of Theorem 2 in [3] and the proof of Theorem 3 in [4]. In both cases, an admissible parameter velocity $\dot{s}_{o}(s), s \in[0,1]$ is first constructed. To show $\dot{s}_{o}(s)$ is a solution to problem $(5)$, the authors actually proved that $\dot{s}_{o}(s)$ is maximum at any parameter value.

The constraints of problem (5) are linear in $a$ and $b$. It will be shown that the nonlinear objective function of problem (5) can be replaced with a linear function in $a$. The idea is to maximize the total feedrate for the end effector of the robots instead of minimizing the traversing time. The feedrate of the end effector of the robot is $F_{d}(s)=\|\mathbf{r} \prime(s)\| \sqrt{a(s)}$, where $\|\mathbf{r} \prime(s)\|=$ $\sqrt{x^{2}(s)+y \prime^{2}(s)+z \prime^{2}(s)}$.

Consider the optimal control problem.

$$
\begin{array}{ll} 
& \max _{b(s)} J_{F}=\int_{0}^{1}\|\mathbf{r} \prime(s)\|^{2} a(s) \mathrm{d} s \\
\text { s.t. } & a \prime(s)=2 b(s), \forall s \in[0,1], \\
& a(s)>0, \forall s \in(0,1), a(0)=a_{0}, a(1)=a_{1}, \\
& \mathbf{m}(s) b+\mathbf{c}(s) a+\mathbf{g}(s)=\tau(s), \\
& \tau_{\min } \leq \tau(s) \leq \tau_{\max } .
\end{array}
$$

Since the objective function of the above problem is linear in $a(s)$, problem (6) is a linear optimal control problem meaning that both the objective function and the constraints are linear in $a$ and $b$. We thus have

Theorem 2. Problem (6) and problem (5) have the same unique optimal solution.

Proof: From [3] and [4], problem (5) has an optimal solution $a_{o}(s)=\dot{s}_{o}(s)^{2}, s \in[0,1]$. Since the two problems have the same constraints, $a_{o}(s)$ is also an admissible pseudo feedrate to problem (6). From Theorem 1, $a_{o}(s)$ achieves maximum values among all $a(s)$ satisfying the constraints of (6) at any parametric value. Since the parametric path $\mathbf{r}(s)$ is assumed to be non-singular, $\|\mathbf{r} /(s)\|^{2}>0$ for all $s \in(0,1)$. Thus $a_{o}(s)$ is also the optimal solution to problem (6). 


\section{Parametrization and Tractable Algorithm for Nominal TMTP}

In this section, a method of parametrization is given to reduce the infinite dimensional optimal control problem (6) to a linear program. The parametrization method will also be used in the next section to solve the robust TMTP.

The first step is to use a special type quadratic B-spline function to approximate the function $a(s)$.

We first define the B-splines used in this paper. Let $K$ and $p$ be positive integers and $s_{i}=\frac{i}{K-p+2}, i=0, \ldots, K-p+2$. Consider the following knot sequence with the first $p+1$ and last $p+1$ knots "clamped".

$$
\mathbf{s}=[\underbrace{0, \ldots, 0}_{p+1}, s_{1}, \ldots, s_{i}, \ldots, s_{K-p+1}, \underbrace{1, \ldots, 1}_{p+1}] .
$$

The B-spline curve of degree $p$ in the parameter interval $[0,1]$ is defined as

$$
\tilde{a}(s)=\sum_{i=0}^{K+1} N_{i, p}(s) \hat{a}_{i},
$$

where $\hat{a}_{i}$ are parameters and $N_{i, p}(s)$ is the $i$-th spline basis function of degree $p$ defined as

$$
\begin{aligned}
N_{i, 0}(s)= & \left\{\begin{array}{cc}
1 & \text { if } \mathbf{s}(i) \leq s<\mathbf{s}(i+1) \\
0 \quad \text { otherwise } & , i=0, \ldots, K+p+1
\end{array}\right. \\
N_{i, k}(s)= & \frac{s-\mathbf{s}(i)}{\mathbf{s}(i+k)-\mathbf{s}(i)} N_{i, k-1}(s)+\frac{\mathbf{s}(i+k+1)-s}{\mathbf{s}(i+k+1)-\mathbf{s}(i+1)} N_{i+1, k-1}(s), \\
& k=1, \ldots, p ; i=0, \ldots, K+p-k+1 .
\end{aligned}
$$

The derivative of the B-spline basis function is given below

$$
N_{i, p}^{\prime}(s)=\frac{\mathrm{d}}{\mathrm{d} s} N_{i, p}(s)=\frac{p}{\mathbf{s}(i+p)-\mathbf{s}(i)} N_{i, p-1}(s)-\frac{p}{\mathbf{s}(i+p+1)-\mathbf{s}(i+1)} N_{i+1, p-1}(s) .
$$

Now, function $a(s)$ is approximated by a quadratic B-spline function $a(s) \approx \tilde{a}(s)$ where $\tilde{a}(s)$ is from (8) for $p=2$. Set $\hat{a}_{0}=a_{0}, \hat{a}_{K+1}=a_{1}$. Due to the multiple knots in the B-splines, we have have $\tilde{a}(0)=\hat{a}_{0}$ and $\tilde{a}(1)=\hat{a}_{K+1}[18]$. The control points $\mathbf{X}=\left[\hat{a}_{1}, \ldots, \hat{a}_{K}\right]^{T}$ will be optimized. The variable $b(s)$ is

$$
b(s)=a \prime(s) / 2 \approx \frac{1}{2} \sum_{i=0}^{K+1} N_{i, p}^{\prime}(s) \hat{a}_{i},
$$

where $N_{i, p}^{\prime}(s)$ is given in (9).

The second step is to reduce the continuous optimal control problem (6) to a finite state optimal problem by using pointwise constraints to replace the continuous torque constraints. The constraint knot points are

$$
\bar{s}_{j}=\left\{\begin{array}{l}
\frac{1}{2} s_{1}, j=1 \\
\frac{1}{2}\left(s_{j-1}+s_{j}\right), j=2, \ldots, K-1 \\
\frac{1}{2}\left(s_{K-1}+1\right), j=K
\end{array}\right.
$$

With these knot points, the objective function can be approximated as

$$
J_{F}=\int_{0}^{1}\|\mathbf{r} \prime(s)\|^{2} a(s) \mathrm{d} s \approx \mathbf{B X}
$$


where $\mathbf{B}=\left(B_{1}, \ldots, B_{K}\right)$ with $B_{i}=\sum_{j=1}^{K}\left\|\mathbf{r} \prime\left(\bar{s}_{j}\right)\right\|^{2} N_{i, p}\left(\bar{s}_{j}\right) \Delta \bar{s}_{j}$ for $\Delta \bar{s}_{j}=s_{j}-s_{j-1}=1 / K$.

From (4), (8), and (10), the torques of the $l$-th joint at the knot points are

$$
\hat{\tau}_{l}=\mathbf{A}_{l} \mathbf{X}+\hat{\mathbf{g}}_{l}, l=1, \ldots, n,
$$

where $\hat{\tau}_{l}=\left[\tau_{l}\left(\bar{s}_{1}\right), \ldots, \tau_{l}\left(\bar{s}_{K}\right)\right]^{T}$ with $\tau_{l}\left(\bar{s}_{j}\right)=\frac{1}{2} m_{l}\left(\bar{s}_{j}\right) \sum_{i=0}^{K+1} N_{i, p}^{\prime}\left(\bar{s}_{j}\right) \hat{a}_{i}+c_{l}\left(\bar{s}_{j}\right) \sum_{i=0}^{K+1} N_{i, p}\left(\bar{s}_{j}\right)$ $\hat{a}_{i}+g_{l}\left(\bar{s}_{j}\right), \mathbf{A}_{l} \in \mathbf{R}^{K \times K}$ with $A_{l j i}=\frac{1}{2} m_{l}\left(\bar{s}_{j}\right) N_{i, p}^{\prime}\left(\bar{s}_{j}\right)+c_{l}\left(\bar{s}_{j}\right) N_{i, p}\left(\bar{s}_{j}\right)$, and $\hat{\mathbf{g}}_{l}=\left[g_{l}\left(\bar{s}_{1}\right)+\right.$ $\left.\xi_{1}, \ldots, g_{l}\left(\bar{s}_{K}\right)+\xi_{K}\right]^{T}$ with $\xi_{j}=\frac{1}{2} m_{l}\left(\bar{s}_{j}\right)\left(N_{0, p}^{\prime}\left(\bar{s}_{j}\right) a_{0}+N_{K+1, p}^{\prime}\left(\bar{s}_{j}\right) a_{1}\right)+c_{l}\left(\bar{s}_{j}\right)\left(N_{0, p}\left(\bar{s}_{j}\right) a_{0}+\right.$ $\left.N_{K+1, p}\left(\bar{s}_{j}\right) a_{1}\right)$.

With the above parametrization, problem (6) is reduced to the following linear program

$$
\begin{array}{ll} 
& \max _{\mathbf{X}} \mathbf{B X} \\
\text { s.t. } & \tilde{a}\left(\bar{s}_{j}\right)=\mathbf{N}_{j} \mathbf{X}+N_{0, p}\left(\bar{s}_{j}\right) a_{0}+N_{K+1, p}\left(\bar{s}_{j}\right) a_{1}>0, j=1, \ldots, K, \\
& \tau_{l,{ }_{\text {min }}} \mathbf{I} \leq \mathbf{A}_{l} \mathbf{X}+\hat{\mathbf{g}}_{l} \leq \tau_{l,,_{\max }} \mathbf{I}, l=1, \ldots, n,
\end{array}
$$

where $\mathbf{I}=(1, \ldots, 1)^{T} \in \mathbf{R}^{K}, \mathbf{B}$ is from (12), $\mathbf{N}_{j}=\left[N_{1, p}\left(\bar{s}_{j}\right), \ldots, N_{K, p}\left(\bar{s}_{j}\right)\right]$, and $\mathbf{A}_{l}, \hat{\mathbf{g}}_{l}$ are from (13). Note that the boundary conditions $a(0)=\hat{a}_{0}=a_{0}$ and $a(1)=\hat{a}_{K+1}=a_{1}$ are automatically satisfied. Properties of the above problem will be discussed in the next section.

\section{Tractable Algorithm for Robust TMTP}

In this section, we will show that the robust TMTP can be reduced to a linear program by using proper parametrization and methods of robust optimization.

Suppose that the dynamics model (4) has uncertainties and the real dynamics model is

$$
\tau(s)=(\mathbf{m}(s)+\Delta \mathbf{m}(s)) \ddot{s}+(\mathbf{c}(s)+\Delta \mathbf{c}(s)) \dot{s}^{2}+(\mathbf{g}(s)+\Delta \mathbf{g}(s))-\Delta \mathbf{d}(s),
$$

where $\Delta \mathbf{m}(s), \Delta \mathbf{c}(s), \Delta \mathbf{g}(s)$, and $\Delta \mathbf{d}(s)$ are the disturbances for $\mathbf{m}(s), \mathbf{c}(s), \mathbf{g}(s)$, and the torque, respectively. The disturbances are assumed to satisfy the following constraints:

$$
\mathcal{D}(\Delta \mathbf{m}, \Delta \mathbf{c}, \Delta \mathbf{g}, \Delta \mathbf{d}):\left\{\begin{array}{l}
\left|\Delta m_{l}(s)\right| \leq B_{m}(l, s), l=1, \ldots, n \\
\left|\Delta c_{l}(s)\right| \leq B_{c}(l, s), l=1, \ldots, n \\
\left|\Delta g_{l}(s)\right| \leq B_{g}(l, s), l=1, \ldots, n \\
\left|\Delta d_{l}(s)\right| \leq B_{d}(l, s), l=1, \ldots, n
\end{array}\right\}
$$

where $\Delta m_{l}(s), \Delta c_{l}(s), \Delta g_{l}(s)$, and $\Delta d_{l}(s)$ are the elements of the vectors $\Delta \mathbf{m}(s), \Delta \mathbf{c}(s), \Delta \mathbf{g}(s)$, and $\Delta \mathbf{d}(s)$, respectively.

Using the min-max approach $[12,13]$, the robust TMTP can be formulated as the following bi-level optimization problem:

$$
\begin{aligned}
& \max _{b(s)} J_{F}=\int_{0}^{1}\|\mathbf{r} \mathbf{r}(s)\|^{2} a(s) \mathrm{d} s \\
& \text { s.t. } a \prime(s)=2 b(s), a(s)>0, s \in(0,1), a(0)=a_{0}, a(1)=a_{1}, \\
& \max _{\mathcal{D}(\Delta \mathbf{m}, \Delta \mathbf{c}, \Delta \mathbf{g}, \Delta \mathbf{d})}((\mathbf{m}(s)+\Delta \mathbf{m}(s)) b(s)+(\mathbf{c}(s)+\Delta \mathbf{c}(s)) a(s)+(\mathbf{g}(s)+\Delta \mathbf{g}(s))-\Delta \mathbf{d}(s)) \leq \tau_{\max } \\
& \min _{\mathcal{D}(\Delta \mathbf{m}, \Delta \mathbf{c}, \Delta \mathbf{g}, \Delta \mathbf{d})}((\mathbf{m}(s)+\Delta \mathbf{m}(s)) b(s)+(\mathbf{c}(s)+\Delta \mathbf{c}(s)) a(s)+(\mathbf{g}(s)+\Delta \mathbf{g}(s))-\Delta \mathbf{d}(s)) \geq \tau_{\min }
\end{aligned}
$$

where $\mathcal{D}(\Delta \mathbf{m}, \Delta \mathbf{c}, \Delta \mathbf{g}, \Delta \mathbf{d})$ is defined in (16). 
Using the parametrization procedure presented in Section 3, the robust TMTP problem (17) is reduced to the following bi-level optimization problem

$$
\begin{array}{ll} 
& \max _{\mathbf{X}} \mathbf{B X} \\
\text { s.t. } & \mathbf{N}_{j} \mathbf{X}+N_{0, p}\left(\bar{s}_{j}\right) a_{0}+N_{K+1, p}\left(\bar{s}_{j}\right) a_{1}>0, j=1, \ldots, K \\
& \max \mathbf{A m}_{l, \Delta \mathbf{A} \mathbf{c}_{l}, \Delta \mathbf{g}_{l}, \Delta \mathbf{d}_{l}} \mathbf{A}_{l} \mathbf{X}+\hat{\mathbf{g}}_{l}+\Delta \mathbf{A} \mathbf{m}_{l} \mathbf{Y}+\Delta \mathbf{A} \mathbf{c}_{l} \mathbf{X}+\Delta \hat{\mathbf{g}}_{l}-\Delta \hat{\mathbf{d}}_{l} \leq \tau_{l, \max } \mathbf{I}, \\
& \min ^{\Delta \mathbf{A} \mathbf{m}_{l}, \Delta \mathbf{A} \mathbf{c}_{l}, \Delta \mathbf{g}_{l, \Delta} \Delta \mathbf{d}_{l}} \\
& \mathbf{A}_{l} \mathbf{X}+\hat{\mathbf{g}}_{l}+\Delta \mathbf{A} \mathbf{m}_{l} \mathbf{Y}+\Delta \mathbf{A} \mathbf{c}_{l} \mathbf{X}+\Delta \hat{\mathbf{g}}_{l}-\Delta \hat{\mathbf{d}}_{l} \geq \tau_{l, \min } \mathbf{I}, \\
& =1, \ldots, n,
\end{array}
$$

where $\mathbf{Y}=\left[\mathbf{N}_{1}^{\prime}, \ldots, \mathbf{N}_{K}^{\prime}\right]^{T} \mathbf{X}$ is a newly defined variable with $\mathbf{N}_{j}^{\prime}=\left[N_{1, p}^{\prime}\left(\bar{s}_{j}\right), \ldots, N_{K, p}^{\prime}\left(\bar{s}_{j}\right)\right]$, $\mathbf{B}, \mathbf{N}_{j}, \mathbf{A}_{l}$, and $\hat{\mathbf{g}}_{l}$ are the same as in (14), $\Delta \hat{\mathbf{g}}_{l}=\left[\Delta g_{l}\left(\bar{s}_{1}\right), \ldots, \Delta g_{l}\left(\bar{s}_{K}\right)\right]^{T}$ is the disturbance of the gravitational torque, $\Delta \hat{\mathbf{d}}_{l}=\left[\Delta d_{l}\left(\bar{s}_{1}\right), \ldots, \Delta d_{l}\left(\bar{s}_{K}\right)\right]^{T}$ is the torque disturbances, $\Delta \mathbf{A m}_{l} \in$ $\mathbf{R}^{K \times K}$ and $\Delta \mathbf{A} \mathbf{c}_{l} \in \mathbf{R}^{K \times K}$ are the uncertainty matrixes of the $l$-th joint with elements $\Delta A m_{l j i}=$ $\frac{1}{2} \Delta m_{l}\left(\bar{s}_{j}\right)$ and $\Delta A c_{l j i}=\Delta c_{l}\left(\bar{s}_{j}\right) N_{i, p}\left(\bar{s}_{j}\right)$ respectively. And the uncertainty constraints are

$$
\begin{aligned}
\Delta \mathbf{A m}_{l} & :\left|\Delta m_{l}\left(\bar{s}_{j}\right)\right| \leq B_{m}\left(l, \bar{s}_{j}\right), \\
\Delta \mathbf{A c}_{l} & :\left|\Delta c_{l}\left(\bar{s}_{j}\right)\right| \leq B_{c}\left(l, \bar{s}_{j}\right), \\
\Delta \mathbf{g}_{l} & :\left|\Delta g_{l}\left(\bar{s}_{j}\right)\right| \leq B_{g}\left(l, \bar{s}_{j}\right), \\
\Delta \mathbf{d}_{l} & :\left|\Delta d_{l}\left(\bar{s}_{j}\right)\right| \leq B_{d}\left(l, \bar{s}_{j}\right), j=1, \ldots, K .
\end{aligned}
$$

Based on a result of Soyster about robust optimization [19], the bi-level optimization problem (18) can be relaxed to the following linear program

$$
\begin{array}{ll} 
& \max _{\mathbf{X}, \mathbf{Z}_{\mathbf{1}}, \mathbf{Z}_{\mathbf{2}}} \mathbf{B X} \\
\text { s.t. } & \mathbf{N}_{j} \mathbf{X}+N_{0, p}\left(\bar{s}_{j}\right) a_{0}+N_{K+1, p}\left(\bar{s}_{j}\right) a_{1}>0, j=1, \ldots, K, \\
& \mathbf{A}_{l} \mathbf{X}+\hat{\mathbf{g}}_{l}+\Delta \mathbf{B} \mathbf{m}_{l} \mathbf{Z}_{\mathbf{1}}+\Delta \mathbf{B} \mathbf{c}_{l} \mathbf{Z}_{\mathbf{2}}+\Delta \mathbf{B}_{g l}+\Delta \mathbf{B}_{d l} \leq \tau_{l,{ }_{\max }} \mathbf{I}, \\
& \mathbf{A}_{l} \mathbf{X}+\hat{\mathbf{g}}_{l}-\Delta \mathbf{B} \mathbf{m}_{l} \mathbf{Z}_{\mathbf{1}}-\Delta \mathbf{B} \mathbf{c}_{l} \mathbf{Z}_{\mathbf{2}}-\Delta \mathbf{B}_{g l}-\Delta \mathbf{B}_{d l} \geq \tau_{l, \min } \mathbf{I}, \\
& -\mathbf{Z}_{\mathbf{2}} \leq \mathbf{X} \leq \mathbf{Z}_{\mathbf{2}},-\mathbf{Z}_{\mathbf{1}} \leq \mathbf{Y} \leq \mathbf{Z}_{\mathbf{1}}, \mathbf{Z}_{\mathbf{1}} \geq \mathbf{0}, \mathbf{Z}_{\mathbf{2}} \geq \mathbf{0}, \\
& \mathbf{Y}=\left[\mathbf{N}_{1}^{\prime}, \ldots, \mathbf{N}_{K}^{\prime}\right]^{T} \mathbf{X}, \\
& l=1, \ldots, n,
\end{array}
$$

where $\mathbf{Z}_{1} \in \mathbf{R}^{K}$ and $\mathbf{Z}_{2} \in \mathbf{R}^{K}$ are new sets of variables, $\Delta \mathbf{B} \mathbf{m}_{l} \in \mathbf{R}^{K \times K}$ and $\Delta \mathbf{B} \mathbf{c}_{l} \in \mathbf{R}^{K \times K}$ are matrixes with elements $\Delta B m_{l j i}=\frac{1}{2} B_{m}\left(l, \bar{s}_{j}\right)$ and $\Delta B c_{l j i}=N_{i, p}\left(\bar{s}_{j}\right) B_{c}\left(l, \bar{s}_{j}\right)$ respectively, $\Delta \mathbf{B}_{g l}=\left[B_{g}\left(l, \bar{s}_{1}\right), \ldots, B_{g}\left(l, \bar{s}_{K}\right)\right]^{T}$, and $\Delta \mathbf{B}_{d l}=\left[B_{d}\left(l, \bar{s}_{1}\right), \ldots, B_{d}\left(l, \bar{s}_{K}\right)\right]^{T}$.

The number of variables in problem $(21)$ is $3 K$ and the number of constraints is $(2 n+7) K$. According to Karmarkar's result [20], problem (21) can be solved with $O\left((n K)^{3.5}\right)$ floating point arithmetic operations.

We briefly discuss the convergency of the method, that is, whether the solutions of the linear programs (14) and (21) convert to the optimal solutions of the optimal control problems (6) and (17), respectively, when $K$ becomes sufficiently large. From the approximation theory, quadratic splines can approximate the solution of optimal control problems to any given precision when $K$ is large enough [21]. Notice that the method of using quadratic splines to approximate the control variable is a special case of the Ritz method, the convergence of which has been proved only for special types of constraints [21-23]. Convergency for problems like the one in this paper seems open. In the next section, numerical results will be used to demonstrate that the method gives the optimal solution with very high precision in less than one second. 


\section{$5 \quad$ Numerical Examples}

The experimental robot is a six-DOF Puma 560 manipulator which is modeled using the Robotics Toolbox for Matlab [24]. The torque limits of the six joints are set to be $[97.6 ; 186.4$; $89.4 ; 24.2 ; 20.1 ; 21.3]$ N.m. Initial and end feedrates of the end effecter are zeros. The path to be traced in the robot work space is shown in Fig.1(a) with the following parametric formula

$$
\left\{\begin{array}{l}
x(s)=0.5 \\
y(s)=0.25\left(\cos (2 a \pi s)-\cos (2 b \pi s)^{3}\right), \quad s \in[0,1] . \\
z(s)=0.25\left(\sin (2 a \pi s)-\sin (2 b \pi s)^{3}\right),
\end{array}\right.
$$

where $a=2, b=1$. The corresponding joint path is shown in Fig.1(b).

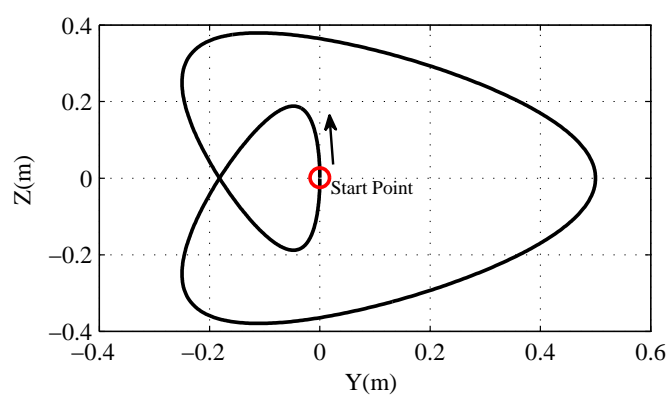

(a)

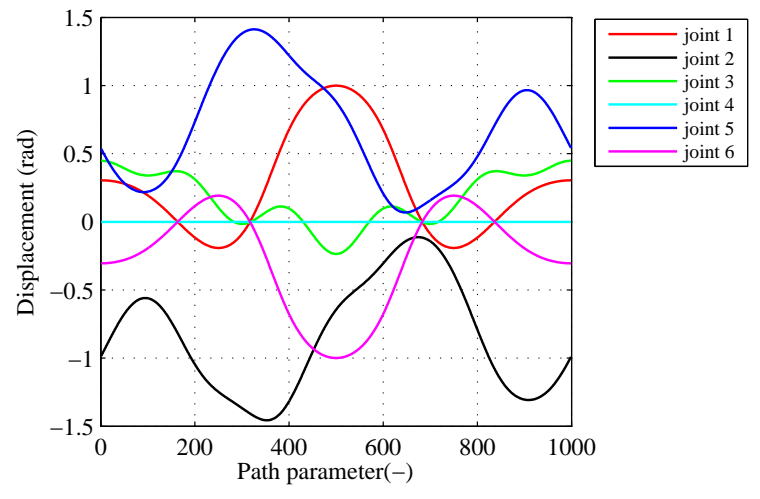

(b)

Figure 1: (a): The task path on Y-Z plane. (b): The corresponding joint path.

First, problem (14) is used to show that the proposed method is efficient and correct. The proposed linear program (LP) approach will be compared to the convex optimization (CP) approach [2] for efficiency. The approach in [2], which is realized by executing the second order cone program (SOCP), was the fastest existing numerical method by now. Both algorithms are implemented by using optimization software package Sedumi [25] in Matlab on a PC with a 2.6 GHz CPU and 2G memory.

Table 1: Comparison of computation times for different $K$

\begin{tabular}{ccccc}
\hline \multirow{2}{*}{ Number $K$} & \multicolumn{2}{c}{ CPU time $(s)$} & \multicolumn{2}{c}{ Optimal motion time $(s)$} \\
\cline { 2 - 5 } & LP & CP in $[2]$ & LP & CP in $[2]$ \\
\hline 100 & 0.3964 & 1.2460 & 1.6473 & 1.6449 \\
\hline 200 & 0.6830 & 2.2970 & 1.6495 & 1.6491 \\
\hline 500 & 0.4630 & 3.6260 & 1.6506 & 1.6506 \\
\hline 700 & 0.9048 & 4.2620 & 1.6508 & 1.6507 \\
\hline 1000 & 0.9300 & 3.4430 & 1.6508 & 1.6508 \\
\hline 2000 & 1.1960 & 6.3440 & 1.6508 & 1.6508 \\
\hline
\end{tabular}




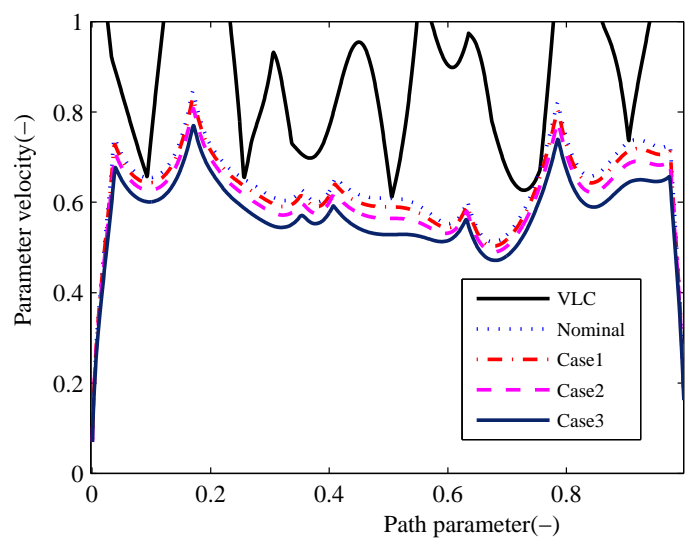

Figure 2: Optimized parameter velocity curves of all four cases for $K=1000$.
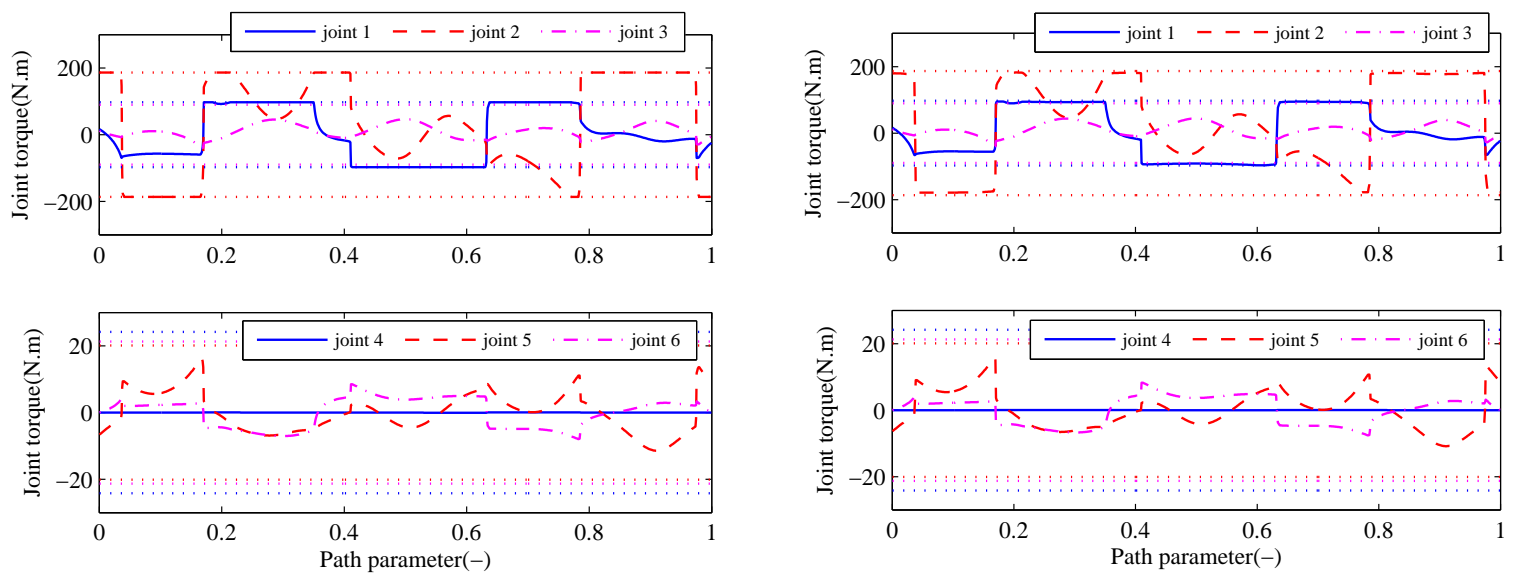

Figure 3: Joint torques of the planned nominal minimum time motion for $K=1000$.

Figure 4: Joint torques of robust minimum time motion in case 1 for $K=1000$.

In Table 1, the computation time and the robot motion time are given for different values of $K$. From Table 1, we can see that the optimal motion times based on formulation (6) and formulation (5) are almost the same, which is consistent with Theorem 2. However, the computation time of our approach is faster than that of [2]. Also note that the actually computational complexity of our approach is sub-linear, meaning less than $O(K)$, which might be due to the special structure of $\mathbf{A}_{l}$ in (14). The practical computational complexity of this approach is better than the complexity $O\left(K^{2}\right)$ of the method given in [11].

The planned joint torques are given in Fig. 3. From [3], the optimal solution of problem (5) is bang-bang-singular in the sense that for any $s \in[0,1]$, the torque of at least one joint reaches its boundary values in the constraint $\tau_{\min } \leq \tau(s) \leq \tau_{\max }$. This property is often used to verify whether a numerical solution is the optimal solution and whether $K$ is large enough. In Fig. 3 , the torque is clearly bang-bang-singular: the torques of joints $2,1,2,1$, and 2 reach their maximal or minimal values in the intervals $[0,0.17],[0.17,0.35],[0.35,0.41],[0.41,0.78],[0.78,1]$, respectively. This indicates that for $K=1000$, the solution obtained with our method most probably gives high precision approximation to the optimal solution of the original problem (5).

Now, it will be shown that the solution of problem (21) is indeed robust. Suppose that the payload holden by the end effector is the only uncertain parameter. As mentioned in [24], the maximum payload capability of the Puma 560 is $2.5 \mathrm{~kg}$. In this section, the robust trajectory 

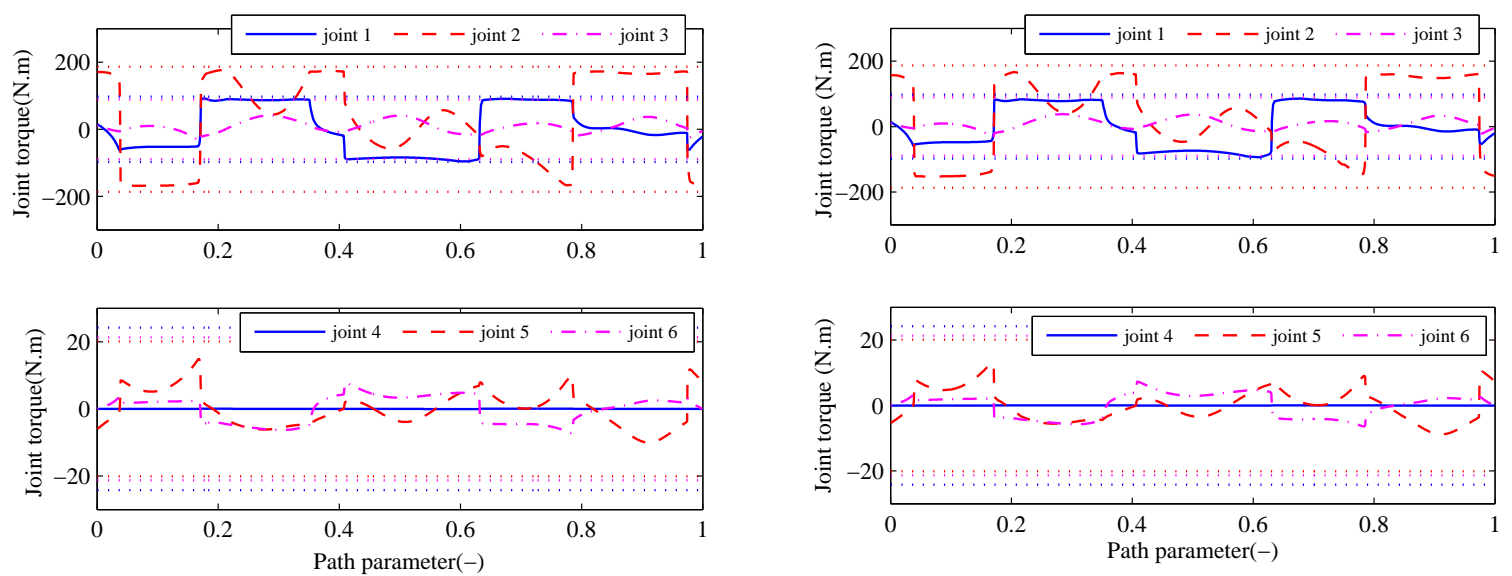

Figure 5: Joint torques of robust minimum time motion in case 2 for $K=1000$.

Figure 6: Joint torques of robust minimum time motion in case 3 for $K=1000$.

planning is implemented under three cases of maximum payload uncertainty assumptions, respectively. The assumptions are $0.5 \mathrm{~kg}$ for case $1,1.25 \mathrm{~kg}$ for case 2 , and $2.5 \mathrm{~kg}$ for case 3 . The disturbed $\Delta \mathbf{m}, \Delta \mathbf{c}$, and $\Delta \mathbf{g}$ are estimated by using the dynamics computation function of the Robotic Toolbox for Matlab.

The computation performance of the proposed robust trajectory planning algorithm is tested by implementing case 2 under different values of $K$. The results are listed in Table 2. From this table, we can see that the calculated optimal solution is convergent when $K$ increases. Also in this example, the actually computational complexity of our approach is less than $O(K)$.

Table 2: Computation performance of robust algorithm for Case 2 with different $K$

\begin{tabular}{ccccccc}
\hline & \multicolumn{7}{c}{ Number $(K)$} \\
\cline { 2 - 7 } & 100 & 200 & 500 & 700 & 1000 & 2000 \\
\hline CPU time & 0.4330 & 0.7080 & 1.0720 & 1.1290 & 1.6920 & 2.6680 \\
\hline Optimal motion time & 1.7355 & 1.7395 & 1.7407 & 1.7408 & 1.7409 & 1.7409 \\
\hline
\end{tabular}

The planned joint torques of the robust minimum time motions in case 1 , case 2 , and case 3 are given in Fig. 4, Fig. 5, and 6, respectively. The optimized parameter velocities $\dot{s}$ for all four cases are shown in Fig. 2. Comparing to Fig. 3, the torques for the robust time-minimum motion are reduced to reserve enough space to reject uncertainties and disturbances without causing torque saturation.

To demonstrate the robustness of the obtained trajectories compared to the nominal one, consider 10 equidistantly distributed mass values of payload in $[0,2.5] \mathrm{kg}$. The planned feedrates in the cases of Fig. 3, Fig. 4, Fig. 5, and Fig. 6 are used to traverse the path, and the corresponding torques are given in Fig. 7, Fig. 8, Fig. 9, and Fig. 10, respectively.

Note that the torque bounds are $[97.6 ; 186.4 ; 89.4 ; 24.2 ; 20.1 ; 21.3]$ N.m. From Fig. 7, Fig. 8 and Fig. 9, we can see that the actual torques pass the given bounds for about $25 \%, 19 \%$ and $11 \%$, respectively. From Fig. 10, it can be seen that the actual torques are all within the given bound, which is consistent with the fact that the robust trajectory in this case is planned under the condition of maximum $2.5 \mathrm{~kg}$ payload.

The robust trajectories for the three cases of uncertainty assumptions are used to traverse 

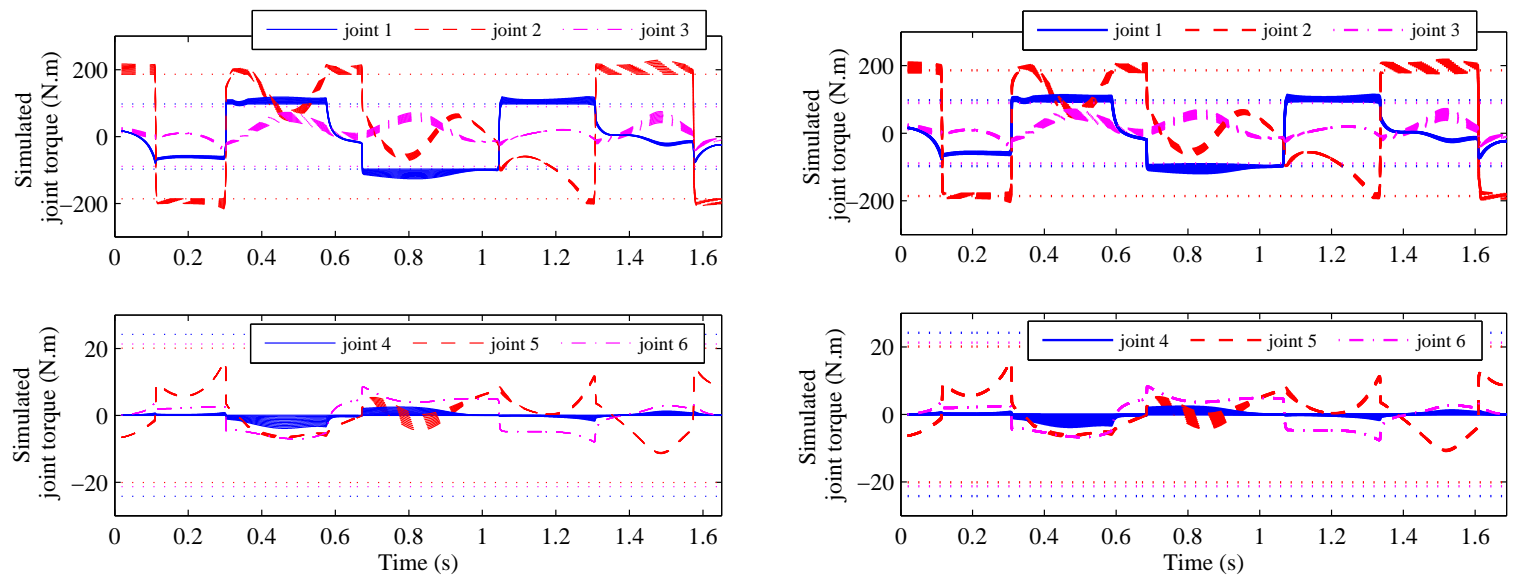

Figure 7: Joint torques for 10 difference payload masses along the nominal reference trajectory.

Figure 8: Joint torques for 10 difference payload masses along the robust reference trajectory of case 1.
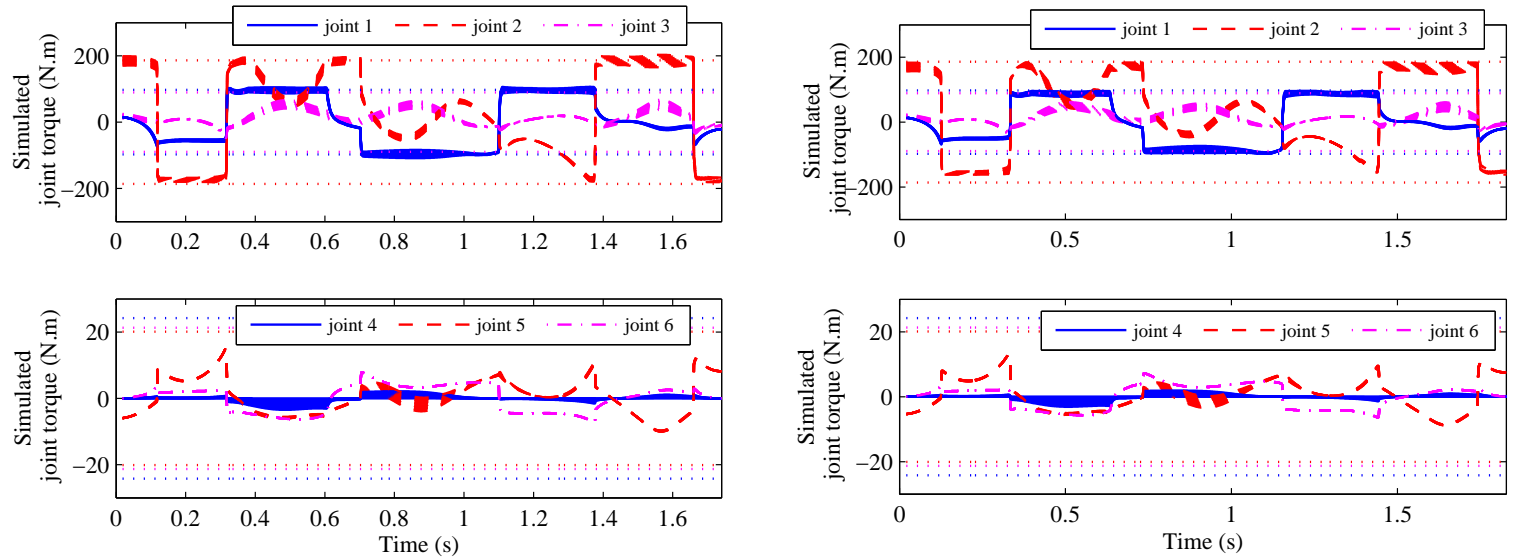

Figure 9: Joint torques for 10 difference payload masses along the robust reference trajectory of case 2 .

Figure 10: Joint torques for 10 difference payload masses along the robust reference trajectory of case 3 .

the path using the ten values of the payload. Detailed data of the experiments are given in Table 3, where Ctime stands for the computation time, Mtime stands for the optimal motion time, RTI stands for the rate of time increase compared with the nominal case, MTV stands for the maximum torque violation, ATV stand for the average torque violation, and RTV stands for the rate of torque violation.

From Table 3, we can see that when the $2.5 \mathrm{~kg}$ payload uncertainty assumption is used, along the generated robust trajectory, the actual torques do not pass the given bounds. By contrast, along the non robust nominal trajectories, at almost all times at least one actual torque passes the given bound and the maximum torque violation reaches $25 \%$ of the given bound. The optimal motion time of this robust trajectory increases about 10.7\% compared with the nominal case, which is a reasonable price to pay for robustness. 
Table 3: Results of trajectory robustness test for $K=1000$

\begin{tabular}{ccccccc}
\hline & Ctime(s) & Mtime $(\mathrm{s})$ & RTI $(\%)$ & MTV(N.m) & ATV(N.m) & RTV $(\%)$ \\
\hline Nominal & 1.4508 & 1.6508 & 0 & 46.39 & 3.99 & 99.16 \\
\hline Case 1 & 1.9810 & 1.6872 & 2.2 & 35.65 & 2.87 & 98.96 \\
\hline Case 2 & 1.6920 & 1.7409 & 5.5 & 21.04 & 1.75 & 82.35 \\
\hline Case 3 & 1.8600 & 1.8281 & 10.7 & None & None & 0 \\
\hline
\end{tabular}

\section{Conclusions}

In this paper, a linear program based robust time minimum trajectory planning approach is presented. The robust version of the TMTP problem is constructed to overcome the parameter uncertainties and torque disturbances of the dynamic system. To make our approach tractable, the TMTP problem is proven to be equivalent to a linear optimal control problem by considering the total motion feedrate of the end-effector of the robot as the objective function. Quadratic B-spline curves are used to parameterize the linear optimal control problem into a linear program which can be solved in polynomial time. The approach theoretically ensures that the generated velocity is time optimal and for every possible realization of the uncertainties and disturbances within the bound, the robust trajectory will not violate any joint torque constraints. Though the proposed algorithm is designed to execute offline, online use is also possible because of the good computation performance.

\section{Acknowledgment}

This research was partially supported by a National Key Basic Research Project of China (2011CB302400), a grant from NSFC (60821002) and a grant from UPC (120501A).

\section{Bibliography}

[1] Katzschmann, R.; Kroger, T.; Asfour, T.; Khatib O.(2013); Towards Online Trajectory Generation Considering Robot Dynamics and Torque Limits, in Intelligent Robots and Systems (IROS), 2013 IEEE/RSJ International Conference on, ISSN 2153-0858, Tokyo, $5644-5651$.

[2] Verscheure, D.; Demeulenaere, B.; Swevers, J.; De Schutter, J.; Diehl, M.(2009); Timeoptimal path tracking for robots: a convex optimization approach, IEEE Trans. on Automatic Control, ISSN 0018-9286, 54(10): 2318-2327.

[3] Bobrow, J.E.; Dubowsky, S.; Gibson, J.(1985); Time-optimal control of robotic manipulators along specified paths, International Journal of Robotics Research, ISSN 0278-3649, 4(3): 3-17.

[4] Shin, K.; McKay, N.(1985); Minimum-time control of robotic manipulators with geometric path constraints, IEEE Trans. on Automatic Control, ISSN 0018-9286, 30(6): 531-541.

[5] Timar, S.D.; Farouki, R.T.(2007); Time-optimal traversal of curved paths by Cartesian CNC machines under both constant and speed-dependent axis acceleration bounds, Robotics and Computer-Integrated Manufacturing, ISSN 0736-5845, 23(5): 563-579. 
[6] Yuan, C.; Zhang, K.; Fan, W.(2013); Time-optimal Interpolation for CNC Machining along Curved Tool Pathes with Confined Chord Error, Journal of Systems Science and Complexity, ISSN 1559-7067, 26(5): 836-870.

[7] Chen, Y.; Desrochers, A.A.(1989); Structure of minimum-time control law for robotic manipulators with constrained paths, in Robotics and Automation, IEEE International Conference on, ISBN 0-8186-1938-4, Scottsdale, USA, 971-976.

[8] Guo, J.X.; Zhang, Q.; Gao, X.S.(2013); Tracking Error Reduction in CNC Machining by Reshaping the Kinematic Trajectory, Journal of Systems Science and Complexity, ISSN 1559-7067, 26(5), 800-817.

[9] Zhang, K.; Yuan, C.M.; Gao, X.S.(2013); Efficient algorithm for feedrate planning and smoothing with confined chord error and acceleration for each axis, The International Journal of Advanced Manufacturing Technology, ISSN 0268-3768 , 66(9): 1685-1697.

[10] Ardeshiri, T.; Norrlof, M.; Lofberg, J.; Hansson, A.(2011); Convex optimization approach for time-optimal path tracking of robots with speed dependent constraint, in Proceedings of the 18th IFAC World Congress, ISSN 1474-6670, Milano, Italy, 14648-14653.

[11] Hauser, K.(2013); Fast Interpolation and Time-Optimization on Implicit Contact Submanifolds, in Proceedings of Robotics: Science and Systems, ISSN 2330-765X, Berlin, Germany.

[12] Shin, K.G.; McKay, N.D.(1987); Robust trajectory planning for robotic manipulators under payload uncertainties, IEEE Trans. on Automatic Control, ISSN 0018-9286, 32(12): 10441054 .

[13] Kieffer, J.; Cahill, A.J.; James, M.R.(1997); Robust and accurate time-optimal pathtracking control for robot manipulators, IEEE Trans. on Robotics and Automation, ISSN 1042-296X, 13(6): 880-890.

[14] Diehl, M.; Gerhard, J.; Marquardt, W.; Monnigmann, M.(2008); Numerical solution approaches for robust nonlinear optimal control problems, Computers \& Chemical Engineering, ISSN 0098-1354, 32(6): 1279-1292.

[15] Marti, K.; Aurnhammer, A.(2002); Robust optimal trajectory planning for robots by stochastic optimization, Mathematical and Computer Modelling of Dynamical Systems, ISSN 1744-5051, 8(1): 75-116.

[16] Chisci, L.; Rossiter, J.A.; Zappa, G.(2001); Systems with persistent disturbances: Predictive control with restrictive constraints, Automatica, ISSN 0005-1098, 37(7): 1019-1028.

[17] Mayne, D.Q.; Seron, M.M.; Rakovic, S.V.(2005); Robust model predictive control of constrained linear systems with bounded disturbances, Automatica, ISSN 0005-1098, 41(2): 219-224.

[18] Patrikalakis, N.M.; Maekawa, T.(2010); Shape Interrogation for Computer Aided Design and Manufacturing, ISBN 978-3-642-04074-0, Springer Berlin, Heidelberg.

[19] Bertsimas, D.; Brown, D.B.; Caramanis, C.(2011); Theory and applications of robust optimization, SIAM Review, ISSN 0036-1445, 53(3): 464-501.

[20] Karmarkar, N.(1984); A new polynomial time algorithm for linear programming, Combinatorica, ISSN 0209-9683, 4(4): 373-395. 
[21] Sirisena, H.R.; Chou, F.S.(1979); Convergence of the control parameterization Ritz method for nonlinear optimal control problems, Journal of Optimization Theory and Applications, ISSN 0022-3239, 29(3): 369-382.

[22] Daniel, J.W.(1973); The Ritz-Galerkin method for abstract optimal control problems, SIAM Journal on Control, ISSN 0036-1402, 11(1): 53-63.

[23] Schwartz, A.L.(1996); Theory and Implementation of Numerical Methods Based on RungeKutta Integration for Solving Optimal Control Problems, Ph.D. Thesis, Univ. of California at Berkeley.

[24] Corke, P.(1996); A robotics toolbox for MATLAB, IEEE Robotics and Automation Magazine, ISSN 1070-9932, 3(1): 24-32.

[25] Sturm, J.F.(1999); Using SeDuMi 1.02, A Matlab toolbox for optimization over symmetric cones, Optimization Methods and Software, ISSN 1055-6788, 11(1-4): 625-653. 